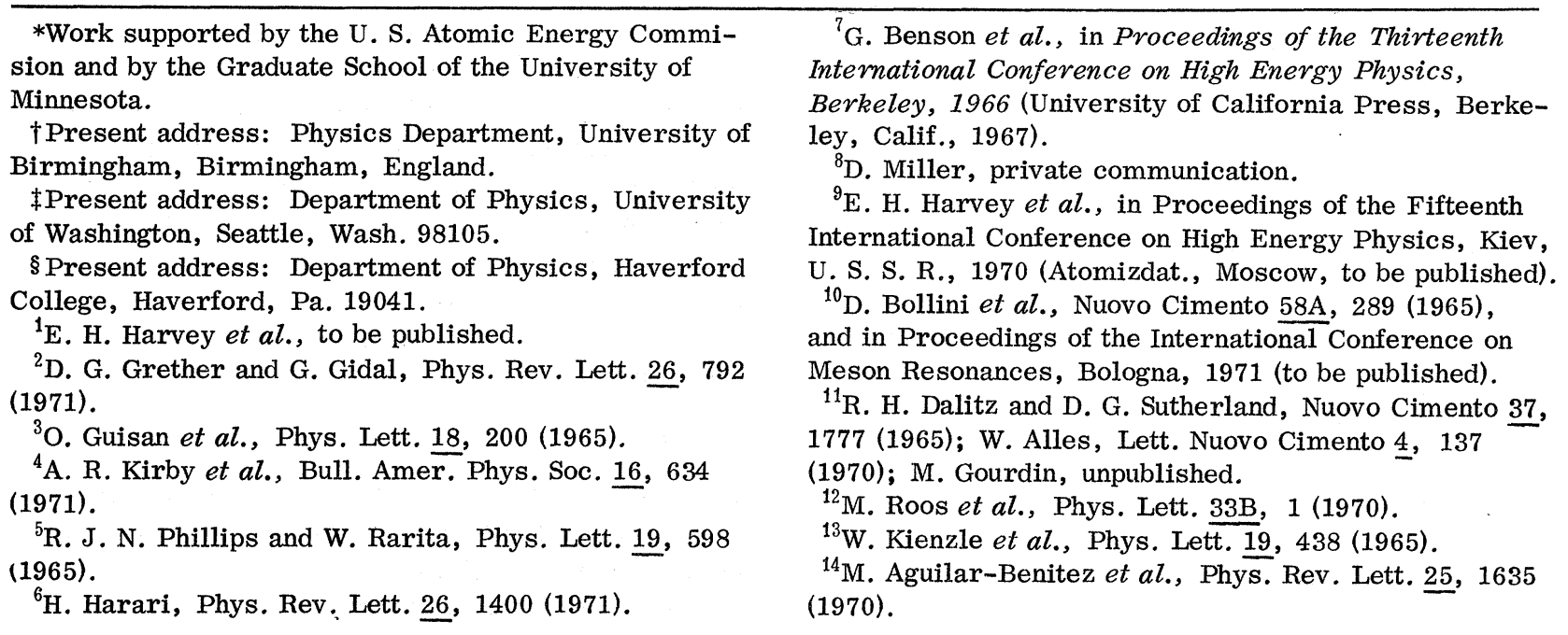

\title{
Precise Determination of $\rho$ - $\omega$ Interference Parameters from Photoproduction of Vector Mesons Off Nucleon and Nuclei
}

\author{
H. Alvensleben, U. Becker, W. Busza, M. Chen, K. J. Cohen, R. T. Edwards, \\ P. M. Mantsch, R. Marshall, T. Nash, M. Rohde, H. F. W. Sadrozinski, \\ G. H. Sanders, H. Schubel, Samuel C. C. Ting, and Sau Lan Wu \\ Department of Physics and Laboratory for Nuclear Science, Massachusetts Institute of Technology, \\ Cambridge, Massachusetts 02139, and Deutsches Elektronen-Synchrotron, DESY, Hamburg, Germany
} (Received 6 July 1971)

\begin{abstract}
We have studied $\rho-\omega$ interference in di-pion photoproduction in the energy range 5-7 $\mathrm{GeV}$ with a total of 630000 pion pairs from hydrogen, carbon, and lead. The mass spectra show clear interference in the vicinity of the $\omega$ mass. A branching ratio $\Gamma_{\omega \rightarrow 2 \pi} /$ $\Gamma_{\omega \rightarrow \mathrm{a} 11}=(1.22 \pm 0.30) \%$ and a relative phase to the $\rho \rightarrow \pi \pi$ amplitude of $96^{\circ} \pm 15^{\circ}$ is found, where the errors include model-dependent uncertainties and are twice the size of the statistical errors alone.
\end{abstract}

The decay $\omega \rightarrow 2 \pi$ does not conserve $G$ parity. The order of magnitude expected for the $\omega \rightarrow 2 \pi$ decay amplitude as compared with the $\rho^{0}-2 \pi$ decay amplitude is the fine-structure constant $\alpha$. Such a result can be visualized as the one-photonexchange mechanism where $\omega \rightarrow \gamma \rightarrow \rho \rightarrow 2 \pi$ with corresponding partial width $\Gamma^{1 / 2}(\omega \rightarrow 2 \pi) \simeq 0.1$ $\mathrm{MeV}^{1 / 2}$, whereas all experiments ${ }^{1}$ to date, though not agreeing with each other, give results larger by an order of magnitude. The large difference between these values is the main motivation for a careful investigation of the $\omega-\rho$ electromagnetic mixing problems.

Previous experiments were done either with a $\pi p$ reaction with a large nonresonant background or with a $\gamma \mathrm{C}$ reaction where one is seriously handicapped by the problem of the nuclear physics of carbon. In contrast to previous measurements, ${ }^{2}$ we have studied $\rho-\omega$ interference using three different targets: hydrogen, carbon, and lead. The analysis of the $\pi^{+} \pi^{-}$spectra from each of these elements poses different advantages. Hydrogen has no complication from nuclear physics but has considerable background. With lead, background and incoherent contributions are small whereas a detailed knowledge of nuclear size is required. Carbon provides something of a compromise. One of the main interests of this experiment was to see if compatible $\omega \rightarrow 2 \pi$ amplitudes could be derived from measurements on such very different nuclei. If so, it would indicate that di-pion photoproduction from nuclei is phenomenologically well understood and is thus a reliable tool for studying $\rho-\omega$ mixing.

In this Letter we communicate a summary of the results. Details of the experiment and of the analysis are being published elsewhere. ${ }^{3}$

The data were taken with a $7.4-\mathrm{GeV}$ brems- 
strahlung beam. The analyzed data cover ranges of $600-900 \mathrm{MeV}$ in di-pion mass $m, 5-7 \mathrm{GeV}$ in di-pion momentum $p$, and $0-0.01 \mathrm{GeV}^{2}$ in fourmomentum transfer $t_{\perp}$. The mass resolution of the spectrometer, crucial to the success of this kind of experiment, was $\approx 8 \mathrm{MeV}$ (full width at half-maximum). The absolute mass calibration and the calculated mass resolution have been verified in a separate experiment ${ }^{4}$ using $\varphi \rightarrow e^{+}$ $+e^{-}$decays. A total of $180000 \pi^{+} \pi^{-}$events were obtained from hydrogen, 375000 from carbon, and 75000 from lead.

The events for each element were binned into an $m, p, t_{\perp}$ grid containing sixty $5-\mathrm{MeV}$ bins in $m$, five $400-\mathrm{MeV}$ in $p$, and five $0.002-\mathrm{GeV}^{2}$ in $t_{\perp}$. The acceptance of each bin was calculated using a Monte Carlo technique. The fit of the theoretical distribution [see Eq. (1) below] to the data was made bin by bin. The measured mass spectra are illustrated in Fig. 1. For graphical representation the data were collapsed in $p$ and $t_{\perp}$ using the observed $p$ and $t_{\perp}$ dependence. All three mass spectra show deviations from a pure $\rho$ shape in the vicinity of the $\omega$ mass, indicating the existence of $\rho-\omega$ interference for all three elements.

To date there is no unambiguous theory which predicts the $\pi^{+} \pi^{-}$spectrum in photoproduction. We fitted the data with two phenomenological forms:

$$
\begin{aligned}
\frac{d \sigma}{d t d m}\left(m, p, t_{\perp}\right) . \\
=2 m R_{i}(m) f_{A}\left(t_{\|}, t_{\perp}\right)+f_{\mathrm{BG}}(m, p), \\
A=\mathrm{H}, \mathrm{C}, \mathrm{Pb},
\end{aligned}
$$

with

$$
\begin{aligned}
& R_{1}(m)=m_{\rho} \Gamma_{\rho}(m) \\
& \quad \times \frac{1}{\pi}\left|\left(\frac{m_{\rho}}{m}\right)^{2} \frac{1}{D_{\rho}(m)}+\xi \frac{e^{i \alpha}}{D_{\omega}(m)}\right|^{2}
\end{aligned}
$$

or

$$
\begin{aligned}
& R_{2}(m)=m_{\rho} \Gamma_{\rho}(m) \frac{1}{\pi} \mid \frac{1}{D_{\rho}(m)} \\
& \quad+\frac{2 F_{A}\left(m_{\rho}^{2}-m^{2}\right)}{m^{2} D_{\rho}(m)}+\left.\frac{\xi e^{i \alpha}}{D_{\omega}(m)}\right|^{2}, \\
& f_{\mathrm{BG}}(m, p)=p^{-2}\left(a m^{2}+b m+c\right), \\
& f_{\mathrm{H}}\left(t_{\|}, t_{\perp}\right)=d \sigma(\gamma p-p \rho) /\left.d t\right|_{t=0} e^{8 t},
\end{aligned}
$$

where $t_{\|}$is the four-momentum transfer for photoproduction of a particle of mass $m$ and momen-
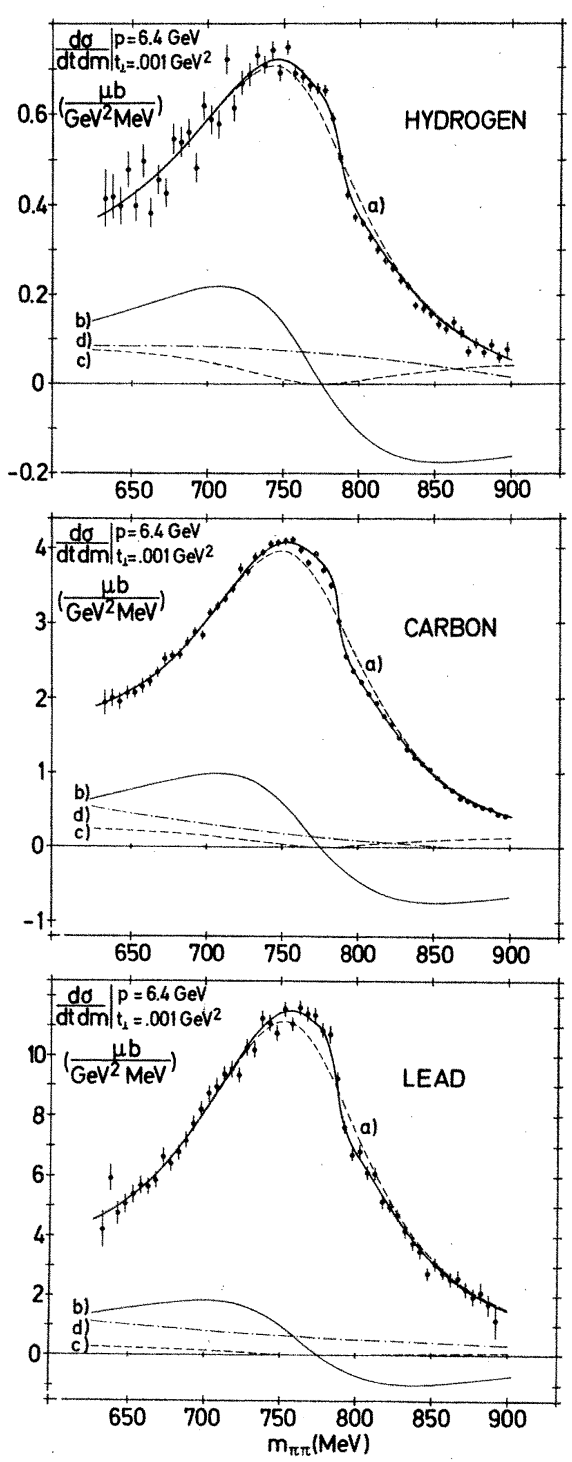

FIG. 1. Cross sections for hydrogen, carbon, and lead. The solid curve is the simultaneous fit to the data using $R_{2}(m)$. The other curves are (a) the same fit with $\xi=0$ (no $\omega$ contribution), (b) the interference with nonresonant $\pi \pi$ amplitude, (c) the nonresonant $\pi \pi$ amplitude squared, and (d) other background.

tum $p$ in the forward direction and $t=t_{\|}+t_{\perp}$. For $f_{\mathrm{C}}$ and $f_{\mathrm{Pb}}$, optical-model calculations were used as described previously, ${ }^{5}$ and $D_{V}(m)=m_{V}{ }^{2}-m^{2}$ $-i m_{V} \Gamma_{V}(m)$

$$
\Gamma_{V}(m)=\Gamma_{V} \frac{m_{V}}{m}\left(\frac{m^{2}-4 m_{\pi}^{2}}{m_{V}{ }^{2}-4 m_{\pi}^{2}}\right)^{3 / 2}, V=\rho, \omega .
$$

$R_{1}(m)$ and $R_{2}(m)$ are mass distributions $s^{6}$ frequently used in the analysis of $\rho$ production with an additional term for $\omega$ production and decay to $\pi \pi$. The relative magnitude and phase of the $\omega \rightarrow \pi \pi$ to 
TABLE I. Results of fits by Eq. (1). Upper part, for individual elements, $\Gamma_{\omega}=11.9 \mathrm{MeV}$; lower part, for $\mathrm{H}, \mathrm{C}$, and $\mathrm{Pb}$ simultaneously.

\begin{tabular}{lc|cccc|ccc}
\hline \hline Element & Fit & $\mathrm{d} \sigma /\left.\mathrm{dt}\right|_{\mathrm{t}=0} ^{\mathrm{nucl}}$ & $\mathrm{m} \rho$ & $\mathrm{m}_{\rho}$ & $\mathrm{m}_{\omega}$ & $\xi$ & $\alpha$ & $\chi^{2} / \mathrm{DF}$ \\
& & $\mu \mathrm{b} /(\mathrm{GeV})^{2}$ & $\mathrm{MeV}$ & $\mathrm{MeV}$ & $\mathrm{MeV}$ & - & degrees & - \\
\hline $\mathrm{H}_{2}$ & $\mathrm{R}_{1}(\mathrm{~m})$ & $113 \pm 5$ & $760 \pm 2$ & $130 \pm 5$ & $783.8 \pm 1.9$ & $.0140 \pm .0014$ & $78 \pm 14$ & 0.89 \\
$\mathrm{C}$ & $\mathrm{R}_{1}(\mathrm{~m})$ & $109 \pm 2$ & $768 \pm 1$ & $140 \pm 2$ & $735.3 \pm 1.1$ & $.0106 \pm .0007$ & $90 \pm 10$ & 1.05 \\
$\mathrm{~Pb}$ & $\mathrm{R}_{1}(\mathrm{~m})$ & $126 \pm 2$ & $781 \pm 2$ & $150 \pm 3$ & $788.8 \pm 1.7$ & $.0121 \pm .0014$ & $106 \pm 15$ & 1.09 \\
& & & & & & & & \\
$\mathrm{H}$ & $\mathrm{R}_{2}(\mathrm{~m})$ & $119 \pm 6$ & $764 \pm 3$ & $136 \pm 5$ & $784.8 \pm 1.9$ & $.0131 \pm .0013$ & $85 \pm 16$ & 0.90 \\
$\mathrm{C}$ & $\mathrm{R}_{2}(\mathrm{~m})$ & $110 \pm 2$ & $769 \pm 2$ & $141 \pm 3$ & $785.7 \pm 1.2$ & $.0105 \pm .0007$ & $92 \pm 10$ & 1.05 \\
$\mathrm{~Pb}$ & $\mathrm{R}_{2}(\mathrm{~m})$ & $128 \pm 3$ & $778 \pm 2$ & $154 \pm 4$ & $787.7 \pm 1.8$ & $.0120 \pm .0013$ & $96 \pm 14$ & 1.09
\end{tabular}

\begin{tabular}{l|llll|lll}
\hline$R_{1}(\mathrm{~m})$ & $115 \pm 1$ & $771 \pm 1$ & $136 \pm 1$ & $787.4 \pm 1.1$ & $.0095 \pm .0005$ & $109 \pm 10$ & $1589 / 1379$ \\
\hline$R_{2}(\mathrm{~m})$ & $125 \pm 1$ & $775 \pm 1$ & $147 \pm 2$ & $787.1 \pm 0.9$ & $.0100 \pm .0005$ & $100 \pm 8$ & $1417 / 1376$ \\
\hline
\end{tabular}

the $\rho \rightarrow \pi \pi$ amplitude are given by $\xi$ and $\alpha$, respectively. $R_{1}$ uses the Ross-Stodolsky factor $\left(m_{\rho} / m\right)^{4}$ to obtain the observed $\rho$ mass shift whereas $R_{2}$ obtains the shift from interference of $\rho$ production with a nonresonant $\pi \pi$ background. ${ }^{6}$

To compare the measured cross sections with Eq. (1), two different methods were used.

(I) To study systematic effects from different nuclei, the fit was applied separately to each element. The free parameters $d \sigma /\left.d t\right|_{t=0}{ }^{\text {nucl }}, m_{\rho}$, $\Gamma_{\rho}, m_{\omega}, \xi, \alpha, a, b, c$, and $F_{A}$ were determined by the fit. The results, with statistical errors, are presented in the upper part of Table I for fits using $R_{1}$ and $R_{2}$ for all three elements. Differences in $\xi$ and $\alpha$ for the two mass-distribution hypotheses could be regarded as systematic errors caused by limitations of the present knowledge of the $\rho \rightarrow \pi \pi$ mass distribution. Variations in $\xi$ and $\alpha$ for the different elements give the size of systematic effects from nuclear physics.

As shown in Table I, all fits are acceptable in terms of $\chi^{2}$ per degree of freedom and no selection between $R_{1}$ and $R_{2}$ is possible with this method. The values of $\xi$ and $\alpha$ obtained are consistent within 2 standard deviations for all three elements, yielding for both distributions $R_{1}$ and $R_{2}$ the same average values of

$$
\xi=0.0113 \pm 0.0006, \quad \alpha=91^{\circ} \pm 7^{\circ} .
$$

Fits were also made in which $m_{\omega}$ was fixed at
785.7 $\mathrm{MeV}$ (an average value from the above fits). A considerably smaller error in $\alpha$ was found. For both $R_{1}$ and $R_{2}$ we obtain $\alpha=91^{\circ} \pm 6^{\circ}$ for hydrogen, $91^{\circ} \pm 4^{\circ}$ for carbon, and $81^{\circ} \pm 6^{\circ}$ for lead. The value of $\xi$ was unchanged.

(II) Since the $\rho$ and $\omega$ parameters are not con-

TABLE II. Sensitivity of $\xi$ and $\alpha$ to deviations from best-fit values.

\begin{tabular}{|c|c|c|c|c|}
\hline changed para & ameter & $\Delta \xi$ & $\Delta \alpha(\mathrm{deg})$ & $\Delta \chi^{2}$ \\
\hline nucl. & $+10 \mu \mathrm{b} / \mathrm{GeV}^{2}$ & - & +7 & +39 \\
\hline$\overline{d t} \quad \prod_{t=0}$ & $-10 \mu \mathrm{b} / \mathrm{GeV}^{2}$ & +.0005 & -15 & +7 \\
\hline \multirow{2}{*}{$\mathrm{m}_{\mathrm{\rho}}$} & $+10 \mathrm{MeV}$ & +.0008 & +17 & +37 \\
\hline & $-10 \mathrm{MeV}$ & +.0005 & -44 & +60 \\
\hline \multirow{2}{*}{$\Gamma_{\rho}$} & $+10 \mathrm{MeV}$ & +.0005 & -3 & +14 \\
\hline & $-10 \mathrm{MeV}$ & -.0008 & +27 & +31 \\
\hline \multirow{2}{*}{$\mathrm{m}_{\omega}$} & $+2 \mathrm{MeV}$ & - & +16 & +5 \\
\hline & - $2 \mathrm{MeV}$ & - & -15 & + \\
\hline \multirow{2}{*}{$\Gamma_{\omega}$} & $+1.8 \mathrm{MeV}$ & +.0011 & - & + \\
\hline & $-1.8 \mathrm{MeV}$ & -.0011 & - & - \\
\hline \multirow{2}{*}{$\Delta \mathrm{m}$} & $+50 \%$ & +.0010 & - & +4 \\
\hline & $-50 \%$ & -.0010 & - & -2.3 \\
\hline \multirow{2}{*}{$B G(m, p)$} & $+50 \%$ & - & -12 & +115 \\
\hline & $-50 \%$ & - & +4 & +113 \\
\hline
\end{tabular}


sistent in the above results, fits have been applied simultaneously to all three elements. In these fits $d \sigma /\left.d t\right|_{t=0} ^{\text {nucl }}, m_{\rho}, \Gamma_{\rho}, m_{\omega}, \xi$, and $\alpha$ were free but were the same for all three elements while the background parameters $a, b, c$, and $F_{A}$ were allowed to be different for the three elements. The results are presented in the lower part of Table I.

For this method, $R_{2}$ is seen to provide a better fit than $R_{1}$. This occurs mainly because of the flexibility of the background parameters $F_{\mathrm{H}}, F_{\mathrm{C}}$, and $F_{\mathrm{Pb}}$ which allow an $A$-dependent mass shift. In the fit using $R_{2}$ we obtain

$$
\xi=0.0100 \pm 0.0007, \quad \alpha=100^{\circ} \pm 7^{\circ} \text {. }
$$

To test the sensitivity of $\chi^{2}, \xi$, and $\alpha$ in this result to changes in the parameters larger than the quoted statistical errors, we have fixed several of the parameters away from their best values. The results are shown in Table II. All other parameters were allowed to vary. From this, one can calculate the best fit for a changed input parameter. The relative deviations observed are of the same size as found in method I. Note also that $\chi^{2}$ increases little for a decreased normalization of $115 \mu \mathrm{b} / \mathrm{GeV}^{2}$.

Since method I ensures the best treatment of the individual elements but does not yield uniform values for the $\rho$ and $\omega$ parameters, whereas method II has the same $\rho$ and $\omega$ parameters but does not indicate $A$-dependent model uncertainties, we consider these methods complementary and quote the average as our best result:

$$
\xi=0.0106 \pm 0.0012, \alpha=96^{\circ} \pm 15^{\circ} .
$$

The errors shown include estimates of systematic errors due to model-dependent uncertainties.

Within the framework of $\rho-\omega$ mass-mixing theo$\mathrm{ry}^{7}$ one can visualize the physical $\rho$ and $\omega$ states as mixtures of pure isospin states of $\left|\rho_{0}\right\rangle,\left|\omega_{0}\right\rangle$ and write $\left.|\rho\rangle=\left|\rho_{0}\right\rangle-\epsilon\left|\omega_{0}\right\rangle, \omega\right\rangle=\epsilon\left|\rho_{0}\right\rangle+\left|\omega_{0}\right\rangle$. The quantities $\alpha$ and $\xi$ determined in this experiment can be related to the branching ratio $R$ for $\omega \rightarrow 2 \pi$ and to the off-diagonal element $\delta=-\left\langle\rho_{0}|M| \omega_{0}\right\rangle$ of the mass matrix. To first order we have ${ }^{1,4}$

$$
\begin{aligned}
\xi & =\left|\frac{\gamma_{\rho}}{\gamma_{\omega}} \frac{A(\omega \omega)}{A(\rho \rho)}\right| \frac{|\delta| 2 m_{\omega}}{\left[\left(m_{\rho}{ }^{2}-m_{\omega}{ }^{2}\right)^{2}+\left(m_{\rho} \Gamma_{\rho}-m_{\omega} \Gamma_{\omega}\right)^{2}\right]^{1 / 2}} \\
& =\left(R \frac{\Gamma_{\omega} m_{\omega}}{\Gamma_{\rho} m_{\rho}}\right)^{1 / 2}\left|\frac{\gamma_{\rho}}{\gamma_{\omega}} \frac{A(\omega \omega)}{A(\rho \rho)}\right| ; \\
\alpha & =\arctan \left(\frac{m_{\rho} \Gamma_{\rho}-m_{\omega} \Gamma_{\omega}}{m_{\rho}{ }^{2}-m_{\omega}{ }^{2}}\right)+\varphi_{p}+\varphi_{\delta} ; \\
\varphi_{p} & =\arg \left(\frac{A(\gamma \omega)}{A(\gamma \rho)}\right),
\end{aligned}
$$

the relative phase of $\omega$ and $\rho$ production;

$$
\varphi_{\delta}=\arg (\delta) ;
$$

where we have used the vector-dominance-model relation $A(\gamma V)=(\alpha \pi)^{1 / 2} / \gamma V A(V V)$, where $A(V V)$ is the scattering amplitude of a vector meson $V$ from a nucleon. Using these formulas and the values $\xi=0.0106 \pm 0.0012, \alpha=96^{\circ} \pm 15^{\circ}, m_{\rho}=770$, $\Gamma_{\rho}=140, m_{\omega}=783.9, \Gamma_{\omega}=11.9 \mathrm{MeV}$, and ${ }^{8} \gamma_{\omega}{ }^{2} / \gamma_{\rho}{ }^{2}$ $=9.4$, we obtain

$$
\begin{aligned}
& R=(1.22 \pm 0.30) \%, \\
& |\delta|=2.09 \pm 0.25 \mathrm{MeV}, \\
& \Gamma_{\omega \rightarrow 2 \pi}=0.145 \pm 0.035 \mathrm{MeV}, \\
& \varphi_{\delta}+\varphi_{p}=-6^{\circ} \pm 15^{\circ} .
\end{aligned}
$$

The errors quoted above are due only to errors in $\xi$ and $\alpha$. The error from $\gamma_{\omega}{ }^{2} / \gamma_{\rho}{ }^{2}=9.4_{-1.6}^{+2.6}$ is not included.

Theoretically, $\delta$ is predicted to be mainly real with a magnitude of the order of the mass difference of the vector mesons. Glashow, ${ }^{9}$ for example, concludes that $\delta \approx-2.7 \mathrm{MeV}$, and several authors ${ }^{7}$ estimate that $\left|\varphi_{\delta}\right| \leqslant 30^{\circ}$.

If we assume that $\left|\varphi_{p}\right|$ is small as indicated by our measurement ${ }^{8}$ on $\gamma+\mathrm{Be} \rightarrow \mathrm{Be}+e^{+}+e^{-}$, and as predicted by the quark model, we see that the results of this experiment fall within the present theoretical estimates.

We are grateful for the support of W. Jentschke, V. F. Weisskopf, P. Demos, A. G. Hill, H. Joos, and $\mathrm{G}$. Weber, who made this collaboration possible. We also thank E. Lohrmann, B. Margolis, and P. J. Biggs for interesting comments, D. Lublow, H. Kumpfert, Mrs. H. Feind, Miss I. Schulz, and Mr. P. Berges for technical assistance.

${ }^{1}$ For a survey of previous experimental work on $\rho-\omega$ interference see G. Goldhaber, in Experimental Meson Spectroscopy, edited by C. Baltay and A. H. Rosenfeld (Columbia U. Press, New York, 1970), p. 59.

${ }^{2}$ P. J. Briggs et al., Phys. Rev. Lett. 24, 1201 (1970); K. C. Moffeit et al., UCRL Report No. UCRL-19753, 1970 (to be published).

${ }^{3} \mathrm{H}$. Alvensleben et al., to be published.

${ }^{4} \mathrm{H}$. Alvensleben et al., DESY Report No. $71 / 26,1971$ (to be published).

${ }^{5}$ K. S. Koelbig and B. Margolis, Nucl. Phys. B6, 85 (1968); H. Alvensleben et al., Nucl. Phys. B18, 333 (1970). The carbon radius was determined from the present data.

${ }^{6}$ G. Kramer and H. R. Quinn, Nucl. Phys. B27, 77 (1971), and references therein; J. Pumplin, Phys. Rev. D $\underline{2}, 1859$ (1970). 
${ }^{7}$ A. S. Goldhaber, G. C. Fox, and C. Quigg, Phys. Lett. 30B, 249 (1969); D. Horn, Phys. Rev. D 1, 1421 (1970); G. R. Allcock, Nucl. Phys. B21, 269 (1970); M. Gourdin, L. Stodolsky, and F. M. Renard, Phys. Lett. 30B, 347 (1969); R. G. Sachs and J. F. Willemsen, Phys. Rev. D 2, 133 (1970).

${ }^{8} \mathrm{H}$. Alvensleben et al., Phys. Rev. Lett. 25, 1373 (1970). [For self-consistency in calculating $\varphi_{\delta}$ a value of $\varphi_{p}=21^{\circ} \pm 20^{\circ}$ should be used rather than the value $\varphi_{\omega \rho}=41^{\circ} \pm 20^{\circ}$. The $20^{\circ}$ difference is an estimate of the effect of $\rho-\omega$ mixing on the process $\gamma+\mathrm{Be} \rightarrow \mathrm{Be}+e^{+}+e^{-}$. See, for example, H. R. Quinn and T. F. Walsh, Nucl. Phys. B22, 637 (1970).] The Daresbury value of $\varphi_{\omega \rho}$ $=118_{-22^{\circ}}^{\circ}$ cannot be reconciled with the present results. See P. J. Briggs et al., Daresbury Nuclear Physics Laboratory Report No. DNPL/P70, 1970 (unpublished).

${ }^{9}$ S. L. Glashow, in Experimental Meson Spectros copy, edited by C. Baltay and A. H. Rosenfeld (Columbia U. Press, New York, 1970), p. 135.

${ }^{10}$ Quinn and Walsh, Ref. 8.

\title{
Hadron Form Factors, Deep-Inelastic Electroproduction, and Massive-Lepton Pair Production: Kinematics or Dynamics?
}

\author{
G. Cocho* and J. J. Salazar $\dagger$ \\ Instituto de Física, Universidad de México, México 20,D.F.
} (Received 28 April 1971)

\begin{abstract}
A theoretical model for the hadrons as extended objects is discussed. The size and shape of hadrons are taken into account by means of an algebraic harmonic-oscillator formalism. This model can explain, with essentially the same parameters, (a) the magnetic form factor of the proton, (b) deep-inelastic electroproduction, and (c) $\mu^{+} \mu^{-}$pair production at high energy. The model is also consistent with the known experimental data on the spacelike and timelike pion form factor.
\end{abstract}

The excited states of the extended hadrons will be described in an algebraic language. In particular we will use unitary representations of the noncompact group $\mathrm{U}(3,1)$. As has been shown elsewhere ${ }^{1}$ the one-rowed representations of such a group contain the same multiplicity of levels as the harmonic oscillator, and the eigenvalues of the invariant operator, which label the representation, may be related to the rms radius of the system and therefore to the "size."

In order to take into account the quantization modes of the "hadronic cavity" one needs an infinite denumerable set of harmonic oscillators. The whole set of these oscillators is included in a single representation of the noncompact group $\mathrm{U}(4,1)$, each oscillator then corresponding to a representation of $\mathrm{U}(3,1)$ which is a subgroup of $\mathrm{U}(4,1)$; therefore, classifying the states by the chain of subgroups $\mathrm{U}(4,1) \supset \mathrm{U}(3,1) \supset \mathrm{U}(3)$ is equivalent to picking up an individual level from a par- ticular oscillator of the set. The states in motion with four-velocity $v_{\mu}=P_{\mu} / m$, where $P_{\mu}$ is the energy-momentum four-vector and $m$ is the mass of the particle, will be labeled by the wave function ${ }^{1}$

$$
\psi(N, d,\{a, b, c\} ; v) .
$$

In order to have unitary representations of $U(4,1)$, $N$ must be real and nonpositive. For each value of $d$ we have an irreducible unitary representation of $U(3,1)$ labeled by the nonpositive number $N-d$. The mass spectrum of the Veneziano-Hagedorn type will then be given by the mass formula

$$
m_{n d}^{2}=m_{0}^{2}+n \Delta_{d}
$$

with $\Delta_{d} \rightarrow \Delta d$ and $\Delta \simeq 1 \mathrm{GeV}^{2}$.

The form factor for the transition (through a scalar interaction) from the ground state of one of these oscillators moving with four-momentum $P_{\mu}$ to any state of this, or any other, oscillator moving with four-momentum $P_{\mu}{ }^{\prime}$ is then given by

$$
\left\langle\psi\left(N, d^{\prime},\left\{a^{\prime}, b^{\prime}, c^{\prime}\right\} ; v^{\prime}\right) \mid \psi(N, d,\{0,0,0\} ; v)\right\rangle=\delta_{d d^{\prime}}\left[\frac{\Gamma(c-N+d)}{c ! \Gamma(-N+d)}\right]^{1 / 2}\left(v \cdot v^{\prime}\right)^{N^{-} d^{-c}}\left(\eta^{\prime} \cdot v\right)^{c}
$$

where the $\eta$ 's are the polarization four-vectors labeling the spin states.

It follows from Ref. 1 that the imaginary part of the amplitude for the Compton-like scattering of 